\title{
Revisar y reescribir textos académicos en la formación del profesorado
}

\section{Check and rewrite academical texts in teachers training}

\author{
Teodoro ÁLVAREZ ANGULO \\ Dpto. Didáctica de la Lengua y la Literatura Facultad de Educación \\ Universidad Complutense de Madrid
}

Recibido: Octubre 2011

Aceptado: Diciembre 2011

\section{Resumen}

Este trabajo refleja una experiencia de aula, y tiene su fundamento en una concepción social, cognitiva, lingüística y didáctica de la expresión escrita de textos académicos. Dicha actividad consiste en la revisión y la reescritura en colaboración del borrador de un texto de divulgación, producido por un grupo de cuatro estudiantes que se forman para ser Maestros de Educación Primaria. El texto es consecuencia de un debate surgido en la clase de Ciencias Naturales, y pretende ser difundido en un planetario. Una vez realizado el borrador del texto, estos estudiantes invitan a todos los compañeros de la clase a revisarlo, en pequeño grupo, con el fin de mejorarlo, mediante la aplicación de una serie de mecanismos cognitivos y de unidades lingüísticas, que se les ofrece como herramientas de ayuda. La proyección didáctica de la experiencia es parte de la formación profesional del profesorado.

Palabras clave: Proceso de escritura. Escritura académica. Revisión del borrador. Reescritura. Texto definitivo. Decir el conocimiento y transformar el conocimiento.

\begin{abstract}
This study identifies a classroom experience from the perspective of social, cognitive, linguistic and didactic concepts of writing academic texts. This involves collaboratively reviewing and rewriting the rough draft of an explanatory text produced by four students who pursue a Degree in Primary Teaching. The text, which is a result of a debate conducted in the 'Natural Sciences' class, is aimed at visitors to a planetarium. To improve the rough draft, the students ask their classmates to review and re-write it in small groups. In doing so, they apply cognitive mechanisms and linguistic units. Primary School Teacher-Training Programmes need to have their students experience didactic sequences.
\end{abstract}

Keywords: Academic writing process. Reviewing and rewriting the rough draft. Final text. Knowledge telling and knowledge transforming. 
Como afirman Mac Arthur, Grahan y Fitzgerald (2006), la escritura es una de las herramientas más poderosas de que dispone el ser humano, en cuanto que nos permite comunicarnos, persuadir a otros y transmitir conocimientos e ideas. Estas atribuciones de la escritura aparecen de manera muy particular en las demandas de escritura de los ámbitos escolares y académicos, mediante la escritura académica.

Por otra parte, la escritura es un fenómeno complejo, ya que integra numerosas habilidades cognitivas (generar ideas; organizarlas de forma coherente; construir oraciones gramaticalmente correctas, adecuadas a un contexto determinado; hacer esquemas y resúmenes; elaborar borradores; manejar un léxico específico y una construcción determinada en cada disciplina; etc.) (Hayes \& Flower, 1980; De Beaugrande, 1984; Bereiter\& Scardamalia, 1984; Grabe \& Kaplan, 1996; Candlin \& Hyland, 1999; Grupo Didactext, 2003; Álvarez, 2010; Carlino, 2006; Castelló, 2007). Ello hace que se deba enseñar a escribir en todas las materias del currículo (Writing Across the Curriculum, WAC; Writing in the Disciplines, WiD), como método de aprendizaje, en cuanto que "la alfabetización académica abarca una amplia gama de prácticas, habilidades e interacciones, que originan el compromiso intelectual con el conocimiento, el pensamiento y el desempeño de la profesión"(Bazerman et al., 2005, p.8). También favorece el dominio de los géneros de la exposición de información, mediante la instrucción y la ejercitación, lo que incrementa la calidad de los textos y el conocimiento científico (escritura epistémica).

Escribir en las materias o asignaturas del currículo (WAC) supone concebir la escritura con finalidades, tales como: (i) aclarar(se) uno mismo conceptos o ideas; (ii) servirse de la escritura como una herramienta de aprendizaje para transformar el conocimiento; (iii) demostrar y transmitir conocimientos; (iv) incrementar el pensamiento y la habilidad de resolver problemas; y (v) vivir en comunidad. Por ello, es preciso determinar las demandas de escritura en cada materia del currículo y en el centro educativo, así como en los ámbitos sociales en que se mueve el individuo en su vida social.

La mejor manera de hacerlo consiste en desentrañar, desde la Educación Primaria, el proceso que se sigue al escribir, de modo que se favorezca el análisis, la reflexión, la interiorización y el automatismo, con el fin de hacer a los alumnos competentes en la habilidad de escribir textos académicos. Esta función de la escritura se pone de manifiesto en las distintas ciencias que lo estudian: psicología cognitiva, pragmática, retórica, lingüística y didáctica de la escritura. Esto mismo lo atestigua el quehacer docente de maestros y profesores en su día a día profesional.

\section{Secuencia didáctica para aprender a escribir textos expositivos}

La propuesta de secuencia didáctica (SD) tiene su fundamento en la explicitud del proceso que conviene seguir para favorecer la reflexión, la interiorización y el dominio de estos textos, los más frecuentes en el ámbito escolar. En este sentido, la SD es una guía o herramienta de la que maestros y profesores pueden servirse para mediar en el 
aprendizaje de la exposición escrita en su propia producción de textos académicos, y de manera particular para facilitar la adquisición y el desarrollo de esta competencia en los escolares de la Educación Obligatoria.

Esta explicitación del proceso de escritura, conforme postulan, entre otros, el Grupo de Didáctica del texto de la Universidad de Ginebra (Dolz, Rosat y Schneuwly, 1990; Schneuwly, 1992); Camps, 2003; Álvarez, 2010; Camargo, Uribe y Caro, 2011), constituye una estrategia de aprendizaje eficaz, ya que tiene en cuenta, por una parte, las categorías cognitivas, "relacionadas con el logro de metas", y, por otra, las metacognitivas, "aquellas que controlan el proceso en sus avances, interferencias o retrocesos" (Grupo Didactext, 2003, p.92).

A tal efecto, la SD se desarrolla en siete fases, con las estrategias cognitivas y metacognitivas que se ponen en marcha en cada una de ellas, y persigue la interiorización, a través de la ejecución, de los distintos pasos que se han de seguir en la elaboración de un texto expositivo. En el cuadro 1 se muestran las distintas fases del proceso de escritura (actividades de los alumnos, mediación de los profesores y producto a que se llega en cada fase), así como la función que cumple la lectura en cada una. El propósito final consiste en hacer a los estudiantes de Magisterio competentes en la destreza de escritura, además de capacitarles para enseñar esta habilidad a alumnos de Educación Primaria.

El planteamiento de escritura en colaboración que se sigue en esta experiencia favorece la integración de las distintas habilidades lingüísticas: hablar para leer; hablar y escuchar para escribir; leer para documentarse; leer para redactar, revisar, reescribir y editar; y escribir y leer para exponer en público, como se muestra en la figura 1. De este modo, la producción de textos en colaboración integra las habilidades lingüísticas y favorece la negociación, el intercambio y el enriquecimiento en los diferentes niveles de la lengua escrita: léxico y semántico, gramatical, pragmático, paratextual, ortográfico, y también para la elección del formato que adoptará el texto y la exposición oral del mismo.

La manera de llevar a cabo la revisión y la reescritura del texto consiste principalmente en trabajar con borradores propios y ajenos, en los que se pueda aplicar las operaciones mentales de manejo de información y las unidades de lengua sobre las que se interviene, teniendo en cuenta las normas de textualidad, los subtipos del texto expositivo y las regularidades lingüísticas de estos textos. Ello posibilita ampliar los conocimientos y transformar el pensamiento. Aquí reside la dificultad de la revisión y la reescritura de textos, tanto se trate de situaciones académicas, profesionales, como de ficción.

\section{Revisar y reescribir textos}

Coincidimos con Millet (2009) en que los aspectos del proceso de escritura menos estudiados son los relativos a la revisión del texto. Probablemente esto tenga que ver con la relación que tiene la escritura con el habla, habilidad en que es imposible la revisión ("The spoken word cannot be revised" (p. 324). Una de las potencialidades del 
trabajo de revisión de textos escritos consiste precisamente en distinguir lo escrito de lo oral; de ahí que los estudiantes terminen asociando la revisión de textos con el hecho de limpiar o pulir el habla ("to clean up speech").

Esto hace que la fase de revisión y reescritura del texto escrito adquiera una especial importancia, además de ser uno de los momentos más fructíferos del aprendizaje, en cuanto que implica una concepción de la escritura como herramienta para adquirir conocimiento y transformar el pensamiento. Esta fase de la producción textual exige una lectura literal, inferencial y crítica, en tanto que pone en juego una serie de conocimientos adquiridos mediante la práctica, como mejorar el texto primigenio, solucionar problemas de escritura, evitar errores y desarrollar estrategias específicas para solventar problemas en los textos. Es asimismo una ocasión para detectar confusiones, tales como las mantenidas entre tecnicismos y el registro culto de la lengua, cuestiones relacionadas con la puntuación, y otras.

En este sentido se puede afirmar que la revisión y la reescritura de textos se basa en la resolución de problemas retóricos. Abordar esta fase de la producción de textos, hasta llegar al texto definitivo, supone reconocer que escribir bien lleva tiempo: tiempo para pensar, tiempo para hacer cambios, tiempo para pulir detalles; en definitiva, tiempo para trabajar las palabras y el texto en su conjunto, antes de que el lector las lea, como afirman Bazerman \& Wiener (2003). Requiere diagnosticar los errores, las incorrecciones, las inexactitudes, las lagunas de información, las repeticiones, las inadecuaciones, etc., y aplicar el o los mecanismos mentales correspondientes y la o las unidades lingüísticas oportunas, así como formular la propuesta de reescritura para mejorar el texto.

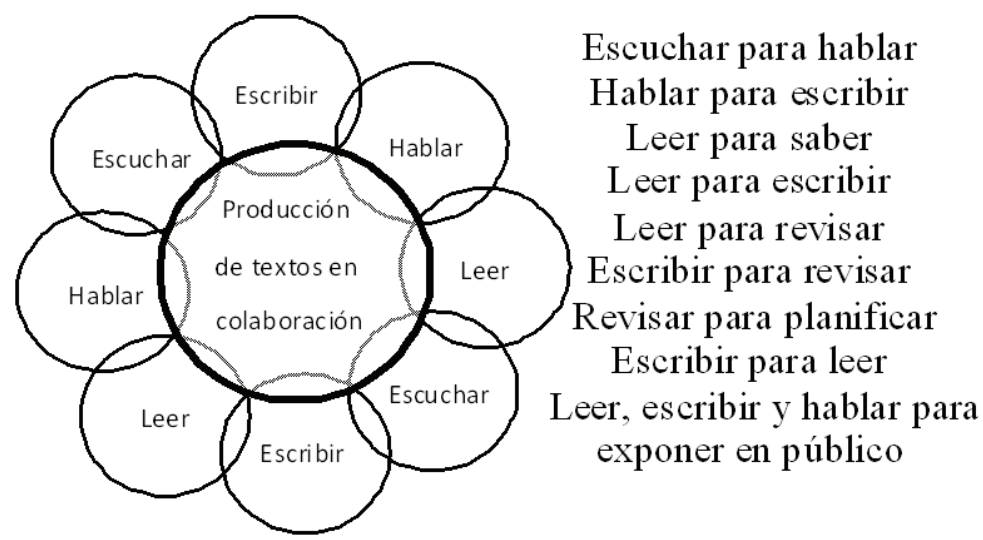

Figura 1. La escritura en colaboración integra las distintas habilidades lingüísticas.

Este planteamiento se basa en los principios que defienden programas o movimientos internacionales, como: Academic Writing, Scafolded Writing and Rewriting in the Disciplines, Writing Across the Curriculum, Writing in Disciplines, Cognitive Process 
in Writing, Research on Writing, Composition Research,Writing to Learn, Writing Communication, Writing in the Community, European Association for Research on Learning and Instruction SIG on Writing, entre otros.

\begin{tabular}{|c|c|c|c|c|c|}
\hline & \multicolumn{4}{|c|}{ PROCESO } & \multirow[b]{2}{*}{ Producto } \\
\hline & Fase & $\begin{array}{c}\text { Función } \\
\text { de la } \\
\text { lectura }\end{array}$ & $\begin{array}{c}\text { Actividades } \\
\text { alumnos }\end{array}$ & $\begin{array}{l}\text { Mediación } \\
\text { profesores }\end{array}$ & \\
\hline $\begin{array}{l}1^{\mathrm{a}} \\
\text { Fase }\end{array}$ & $\begin{array}{l}\text { Contextualización } \\
\text { del proyecto de } \\
\text { escritura. } \\
\text { Qué sabemos } \\
\text { sobre el contexto } \\
\text { (intención, desti- } \\
\text { natario, conoci- } \\
\text { mientos previos } \\
\text { sobre el tema y } \\
\text { sobre el género } \\
\text { discursivo). }\end{array}$ & $\begin{array}{l}\text { Leer el } \\
\text { mundo. }\end{array}$ & $\begin{array}{l}\text { - Intercambian } \\
\text { acerca de los } \\
\text { conocimientos } \\
\text { previos del } \\
\text { contexto de } \\
\text { escritura del } \\
\text { texto } \\
\text { expositivo. } \\
\text { - Hacen un } \\
\text { cartel sobre los } \\
\text { elementos del } \\
\text { contexto, con } \\
\text { la ayuda de la } \\
\text { información } \\
\text { del cuadro } 2 . \\
\text { - Proponen } \\
\text { temas me- } \\
\text { diante lluvia } \\
\text { de ideas y } \\
\text { eligen uno por } \\
\text { consenso. } \\
\text { - Precisan la } \\
\text { intención del } \\
\text { texto que se va } \\
\text { a escribir. } \\
\text { Hacen un } \\
\text { cartel con } \\
\text { posibles inten- } \\
\text { ciones. Se } \\
\text { sirven de la } \\
\text { ayuda del } \\
\text { cuadro3. } \\
\text { - Determinan } \\
\text { el o los desti- } \\
\text { natarios del } \\
\text { texto que se va } \\
\text { a escribir. }\end{array}$ & $\begin{array}{l}\text { - Gestionan el } \\
\text { intercambio. } \\
\text { - Seleccionan el } \\
\text { tema a partir de } \\
\text { haber descu- } \\
\text { bierto y anota- } \\
\text { do los intereses } \\
\text { de los alumnos. } \\
\text { - Concretan las } \\
\text { posibles inten- } \\
\text { ciones: saber } \\
\text { pensar, poder } \\
\text { conocer, saber } \\
\text { explicar a otros, } \\
\text { transmitir, dar } \\
\text { cuenta de lo } \\
\text { que se sabe. } \\
\text { - Identifican a } \\
\text { los posibles } \\
\text { destinatarios: } \\
\text { uno mismo, } \\
\text { compañeros de } \\
\text { la clase o de } \\
\text { otras clases, } \\
\text { intercambio } \\
\text { con otros cen- } \\
\text { tros de la loca- } \\
\text { lidad o de fue- } \\
\text { ra, comunidad } \\
\text { escolar, difu- } \\
\text { sión entre co- } \\
\text { lectivos intere- } \\
\text { sados por el } \\
\text { tema, profesor, } \\
\text { etc. } \\
\text { - Ayudan a } \\
\text { recuperar cono- }\end{array}$ & $\begin{array}{l}\text { Elaboración de } \\
\text { una ficha de } \\
\text { registro en la } \\
\text { que se haga } \\
\text { constar el tema } \\
\text { elegido por } \\
\text { consenso; el } \\
\text { destinatario y la } \\
\text { intención parti- } \\
\text { culares; lo que } \\
\text { se sabe sobre el } \\
\text { tema y lo que se } \\
\text { quiere saber, y } \\
\text { cómo } \\
\text { difundirlo. }\end{array}$ \\
\hline
\end{tabular}




\begin{tabular}{|c|c|c|c|c|c|}
\hline & & & $\begin{array}{l}\text { Hacen un } \\
\text { cartel con } \\
\text { posibles desti- } \\
\text { natarios. Se } \\
\text { sirven de la } \\
\text { ayuda del } \\
\text { cuadro } 4 . \\
\text { - Piensan y } \\
\text { anotan cono- } \\
\text { cimientos } \\
\text { sobre el tema. } \\
\text { Elaboran una } \\
\text { ficha. } \\
\text { - Piensan en } \\
\text { cómo difundir } \\
\text { la informa- } \\
\text { ción. Hacen un } \\
\text { listado de } \\
\text { formas de } \\
\text { difusión. }\end{array}$ & $\begin{array}{l}\text { cimientos de la } \\
\text { memoria y de } \\
\text { la experiencia. } \\
\text { - Ayudan a } \\
\text { elegir el posible } \\
\text { canal de difu- } \\
\text { sión: periódico, } \\
\text { radio, dossier } \\
\text { de documenta- } \\
\text { ción, mural, } \\
\text { etc. }\end{array}$ & \\
\hline $2^{\mathrm{a}}$ & $\begin{array}{l}\text { Necesidades de } \\
\text { documentación y } \\
\text { conocimiento del } \\
\text { género. } \\
\text { Qué queremos } \\
\text { saber sobre el } \\
\text { tema y sobre } \\
\text { cómo lo vamos a } \\
\text { transmitir. }\end{array}$ & $\begin{array}{l}\text { Leer para } \\
\text { saber. }\end{array}$ & $\begin{array}{l}\text { - Expresan } \\
\text { aspectos par- } \\
\text { ciales que se } \\
\text { quieran incor- } \\
\text { porar al tema } \\
\text { global, me- } \\
\text { diante una } \\
\text { lluvia de ideas. } \\
\text { Lo recogen en } \\
\text { la pizarra. } \\
\text { - Mencionan y } \\
\text { seleccionan } \\
\text { fuentes de } \\
\text { información. } \\
\text { - Leen o escu- } \\
\text { chan la infor- } \\
\text { mación, la } \\
\text { seleccionan y } \\
\text { la anotan. } \\
\text { - Registran la } \\
\text { información } \\
\text { obtenida me- } \\
\text { diante esque- } \\
\text { mas, resúme- } \\
\text { nes o fichas } \\
\text { pautadas. }\end{array}$ & $\begin{array}{l}\text { - Guían y ano- } \\
\text { tan las propues- } \\
\text { tas. } \\
\text { - Asesoran } \\
\text { sobre el manejo } \\
\text { de fuentes de } \\
\text { información, } \\
\text { mediante el } \\
\text { diseño y el } \\
\text { seguimiento de } \\
\text { una ficha pau- } \\
\text { tada para la } \\
\text { selección de } \\
\text { información. } \\
\text { - Guían el ma- } \\
\text { nejo de fuentes } \\
\text { documentales y } \\
\text { la selección de } \\
\text { información. } \\
\text { - Ayudan a } \\
\text { seleccionar la } \\
\text { información y a } \\
\text { registrarla. }\end{array}$ & $\begin{array}{l}\text { Fichas cumpli- } \\
\text { mentadas con la } \\
\text { información } \\
\text { seleccionada. } \\
\text { Ver Anexo } 1 .\end{array}$ \\
\hline
\end{tabular}




\begin{tabular}{|c|c|c|c|c|c|}
\hline $3^{a}$ & $\begin{array}{l}\text { Del caos de la } \\
\text { información al } \\
\text { orden del texto. } \\
\text { Cómo se organiza } \\
\text { un texto } \\
\text { expositivo. }\end{array}$ & $\begin{array}{l}\text { Leer para } \\
\text { escribir. }\end{array}$ & $\begin{array}{l}\text { - Relacionan la } \\
\text { información } \\
\text { seleccionada } \\
\text { con los "mol- } \\
\text { des" o subti- } \\
\text { pos de repre- } \\
\text { sentación de la } \\
\text { exposición, } \\
\text { mostrados en } \\
\text { cartulinas por } \\
\text { las paredes del } \\
\text { aula. Ver los } \\
\text { modelos en } \\
\text { Álvarez Angu- } \\
\text { lo, 2005:126- } \\
\text { 129. } \\
\text { - Optan por el } \\
\text { más conve- } \\
\text { niente, en cada } \\
\text { caso. } \\
\text { - Organizan la } \\
\text { información } \\
\text { en subtemas o } \\
\text { epígrafes. }\end{array}$ & $\begin{array}{l}\text { - Explican los } \\
\text { diferentes sub- } \\
\text { tipos de repre- } \\
\text { sentación de la } \\
\text { exposición de } \\
\text { información, } \\
\text { mediante el } \\
\text { diseño de un } \\
\text { cartel con los } \\
\text { diferentes sub- } \\
\text { tipos para ex- } \\
\text { poner en el } \\
\text { aula. } \\
\text { - Explican las } \\
\text { principales } \\
\text { regularidades } \\
\text { del texto. Ver } \\
\text { Álvarez Angu- } \\
\text { lo, 2005:131- } \\
\text { 133. } \\
\text { - Diseñan un } \\
\text { cartel con las } \\
\text { principales } \\
\text { regularidades } \\
\text { lingüísticas y } \\
\text { textuales del } \\
\text { mencionado } \\
\text { texto. } \\
\text { - Guían la deci- } \\
\text { sión de cada } \\
\text { alumno. } \\
\text { - Acompañan a } \\
\text { los alumnos en } \\
\text { la organización } \\
\text { de los subtemas } \\
\text { o epígrafes. }\end{array}$ & $\begin{array}{l}\text { Elaboración de } \\
\text { mapas concep- } \\
\text { tuales, esque- } \\
\text { mas, resúmenes, } \\
\text { etc., con la } \\
\text { información } \\
\text { manejada, orga- } \\
\text { nizada en el } \\
\text { subtipo o molde } \\
\text { correspondiente. }\end{array}$ \\
\hline $4^{a}$ & $\begin{array}{l}\text { Producción del } \\
\text { texto: hacia el } \\
\text { primer borrador. } \\
\text { Qué debemos } \\
\text { tener en cuenta } \\
\text { para producir el } \\
\text { texto. }\end{array}$ & $\begin{array}{l}\text { Leer para } \\
\text { escribir. }\end{array}$ & $\begin{array}{l}\text { - Cotejan y } \\
\text { plasman las } \\
\text { características } \\
\text { lingüísticas y } \\
\text { textuales del } \\
\text { texto } \\
\text { expositivo. } \\
\text { - Escriben } \\
\text { borrador(es) o } \\
\text { texto(s) inter- }\end{array}$ & $\begin{array}{l}\text { - Insisten en la } \\
\text { explicación de } \\
\text { las principales } \\
\text { regularidades } \\
\text { del texto en } \\
\text { cuestión. Dise- } \\
\text { ñan un cartel } \\
\text { con las princi- } \\
\text { pales regulari- } \\
\text { dades lingüísti- }\end{array}$ & $\begin{array}{l}\text { Borradores o } \\
\text { textos interme- } \\
\text { dios. }\end{array}$ \\
\hline
\end{tabular}




\begin{tabular}{|c|c|c|c|c|c|}
\hline & & & medio(s). & $\begin{array}{l}\text { cas y textuales } \\
\text { del mencionado } \\
\text { texto. } \\
\text { - Favorecen la } \\
\text { idea de reescri- } \\
\text { tura dentro del } \\
\text { proceso. }\end{array}$ & \\
\hline $5^{a}$ & $\begin{array}{l}\text { Revisión. Cómo } \\
\text { revisar el texto: } \\
\text { identificar logros } \\
\text { y problemas tex- } \\
\text { tuales y } \\
\text { resolverlos. }\end{array}$ & $\begin{array}{l}\text { Leer para } \\
\text { evaluar y } \\
\text { mejorar }\end{array}$ & $\begin{array}{l}\text { - Utilizan } \\
\text { guías de ayu- } \\
\text { da o listas de } \\
\text { control para } \\
\text { monitorizar la } \\
\text { revisión del } \\
\text { proceso de } \\
\text { producción y } \\
\text { el producto } \\
\text { final. Ver } \\
\text { Anexo 3. } \\
\text { - Analizan } \\
\text { borrador(es) o } \\
\text { texto(s) inter- } \\
\text { medio(s). } \\
\text { - Producen el } \\
\text { texto } \\
\text { definitivo. }\end{array}$ & $\begin{array}{l}\text { - Explican las } \\
\text { funciones de } \\
\text { revisión, } \\
\text { control e ins- } \\
\text { pección como } \\
\text { mecanismos } \\
\text { sociales y lo } \\
\text { relacionan con } \\
\text { la revisión de } \\
\text { textos. } \\
\text { - Guían el pro- } \\
\text { ceso de revisión } \\
\text { (antes, durante } \\
\text { y después) } \\
\text { hasta llegar al } \\
\text { texto final } \\
\text { (producto). Ver } \\
\text { Anexo 2. } \\
\text { - Proporcionan } \\
\text { una guía de } \\
\text { revisión de } \\
\text { textos. } \\
\text { - Guían la pro- } \\
\text { ducción del } \\
\text { texto definitivo }\end{array}$ & $\begin{array}{l}\text { Texto } \\
\text { definitivo. }\end{array}$ \\
\hline $6^{\mathrm{a}}$ & Edición. & $\begin{array}{l}\text { Leer para } \\
\text { reescribir. }\end{array}$ & $\begin{array}{l}\text { - Piensan en } \\
\text { formatos para } \\
\text { editar la in- } \\
\text { formación } \\
\text { contenida en el } \\
\text { texto } \\
\text { definitivo. } \\
\text { - Deciden el } \\
\text { formato más } \\
\text { adecuado a la } \\
\text { situación. }\end{array}$ & $\begin{array}{l}\text {-Asesoran so- } \\
\text { bre posibles } \\
\text { formatos } \\
\text { conforme a la } \\
\text { intención que } \\
\text { se persiga y al } \\
\text { público al que } \\
\text { vaya destinada } \\
\text { la información. }\end{array}$ & $\begin{array}{l}\text { Edición en el } \\
\text { formato corres- } \\
\text { pondiente. }\end{array}$ \\
\hline
\end{tabular}




\begin{tabular}{|l|l|l|l|l|l|}
\hline $7^{\mathrm{a}}$ & $\begin{array}{l}\text { Exposición en } \\
\text { público. }\end{array}$ & $\begin{array}{l}\text { Leer para } \\
\text { hablar en } \\
\text { público. }\end{array}$ & $\begin{array}{l}\text { - Ensayan y } \\
\text { ajustan la } \\
\text { exposición } \\
\text { oral y, en su } \\
\text { caso, la defen- } \\
\text { sa del mismo. }\end{array}$ & $\begin{array}{l}\text { - Escuchan, } \\
\text { ayudan y revi- } \\
\text { san el formato } \\
\text { y la exposición } \\
\text { del texto. }\end{array}$ & $\begin{array}{l}\text { Exposición en } \\
\text { público, y, en } \\
\text { su caso, defen- } \\
\text { sa. }\end{array}$ \\
\hline
\end{tabular}

Cuadro 1. Secuencia didáctica para la producción de textos expositivos

\section{EI texto expositivo y su escritura}

\section{El contexto de la escritura}

Para situar o contextualizar lo que vamos a escribir, es necesario empezar por plantearse estas preguntas: Quién escribe qué a quién, con qué propósito, cuándo, dónde y cómo. ("Lo más importante es no dejar de hacerse preguntas." Albert Einstein).

Cuadro 2. El contexto de la escritura.

\begin{tabular}{|c|}
\hline El texto expositivo y su escritura \\
Principales fines o intenciones del texto \\
Desarrollar el pensamiento \\
Poder conocer y ampliar conocimientos \\
Saber explicar a otro(s) ideas y/o conceptos \\
Dar cuenta de lo que se sabe \\
Despertar el interés por un tema \\
Piensa cuál es tu intención antes de empezar a escribir \\
\hline
\end{tabular}

Cuadro 3. Finalidad de la escritura.

\section{El texto expositivo y su escritura Búsqueda del o de los destinatarios del texto}

- Uno mismo

- Compañeros de clase o de otras clases

- Intercambio con otros centros de la localidad o de fuera

- Comunidad escolar (alumnos, familias, profesorado, personal no docente

- Administración (local, autonómica, central)

- Medios de comunicación

- Colectivos interesados por el tema

- Profesor de la asignatura

- Otros

Antes de empezar a escribir y durante el proceso de escritura, ten presente a la persona o personas a quien(es) va dirigido el texto.

Cuadro 4. Destinatario del texto. 
Estos cuadros, aportados a título de ejemplo, son guías o herramientas para ayudar a construir el contexto del texto que se pretende escribir.

\section{Objetivos}

Esta experiencia persigue los siguientes objetivos:

(i) Poner de manifiesto el proceso de escritura de un texto expositivo o académico, explicitando las distintas fases y aplicando estrategias que favorezcan la reflexión, la interiorización y la automatización de la habilidad de escribir.

(ii) Producir el borrador de un texto académico en colaboración entre cuatro estudiantes de Magisterio, de la asignatura de Didáctica de la Lengua Española, teniendo en cuenta el contexto, el proceso y el texto.

(iii) Implicar a todo el grupo de la clase en la fase de revisión y reescritura del borrador propuesto, con el fin de enriquecerlo, hasta llegar a la edición definitiva del texto, con las aportaciones de todo el grupo de la clase.

(iv) Adquirir el procedimiento de enseñanza y aprendizaje de la escritura académica en la Educación Obligatoria, mediante secuencias didácticas que expliciten el proceso de escritura, en las materias del currículo.

(v) Con todo ello se pretende descubrir la influencia de las oportunidades de reflexión que proporciona la explicitación del contexto, del proceso y del texto, en la composición de textos escritos que llevan a cabo estudiantes universitarios, que se forman para ser Maestros de Educación Primaria, con el fin de que les sirva de modelo para su posterior práctica docente.

\section{Método}

\section{Contexto}

La experiencia de aula llevada a cabo por cuatro estudiantes de segundo año de la titulación de Grado de Magisterio de Educación Primaria, en la asignatura de Didáctica de la Lengua Española, consiste en producir un texto expositivo en colaboración. Se aborda este tipo de texto, el expositivo, por ser el más frecuente en ámbitos académi$\cos$.

El tema sobre el que los estudiantes deciden escribir versa sobre una breve introducción a la terraformación, destinada a un público no especializado: visitantes de un planetario. Es consecuencia de un debate mantenido en la asignatura de Didáctica de las Ciencias Naturales de esta misma titulación.

Para ello, el grupo mencionado sigue las fases de la secuencia didáctica, tal y como se describen más arriba, hasta llegar a la fase de revisión y reescritura, momento en que se invita a participar a todo el grupo de la clase, organizado, a su vez, en grupos pequeños, con la finalidad de mejorar el texto para su posterior edición. 


\section{Actividad de escritura}

El procedimiento que se sigue para ello consiste en producir el texto en colaboración, en grupo pequeño, hasta la fase de elaboración del borrador. Una vez realizado el primer borrador, se invita a toda la clase a revisar el texto (reescribirlo), para, posteriormente, editarlo.

Llegados a la fase de revisión y reescritura del texto, una vez producido el borrador, los estudiantes deciden implicar a sus compañeros de clase y plantean, en dos sesiones, la revisión del texto propuesto para realizarlo en gran grupo.

Para ello, en la primera sesión, explican: (i) el proceso textual desarrollado hasta el momento, conforme al cuadro 5; (ii) las unidades lingüísticas que componen la textualidad (Ver cuadro 5); (iii) los niveles de intervención lingüística y textual de la revisión (Ver cuadro 6); (iv) los principales mecanismos mentales que intervienen en la revisión textual (Ver cuadro 6); (v) la plantilla de ayuda para revisar textos expositivos, en la que hay ítems correspondientes al contexto, al proceso y al texto (Ver Anexo 3).

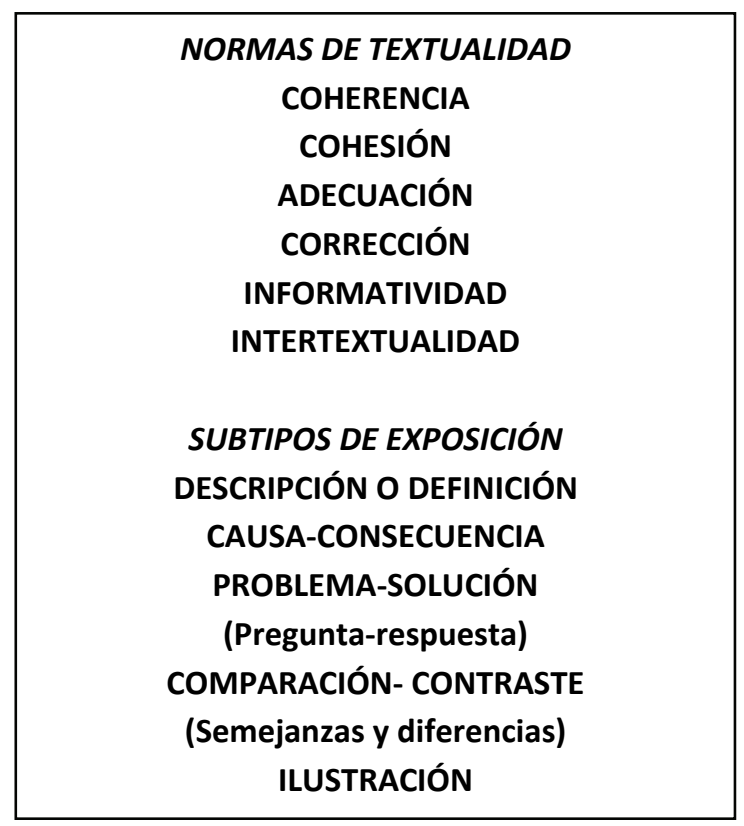

Cuadro 5. Cómo se construye un texto.
REGULARIDADES LINGÜÍSTICAS DEL TEXTO EXPOSITIVO

ORGANIZADORES CONECTORES LÓGICOS TÍTULOS, SUBTÍTULOS Y EPÍGRAFES MARCAS DE EXPLICACIÓN FORMAS VERBALES APOSICIONES EXPLICATIVAS PRECISIÓN LÉXICA Y RIGOR CONTRUCCIÓN LÓGICA DE LA FRASE REFORMULACIONES EJEMPLIFICACIONES ILUSTRACIONES DEFINICIONES, CITAS Y REFERENCIAS DESCRIPCIONES Y COMPARACIONES ENUMERACIONES FÓRMULA DE CIERRE O RESUMEN 

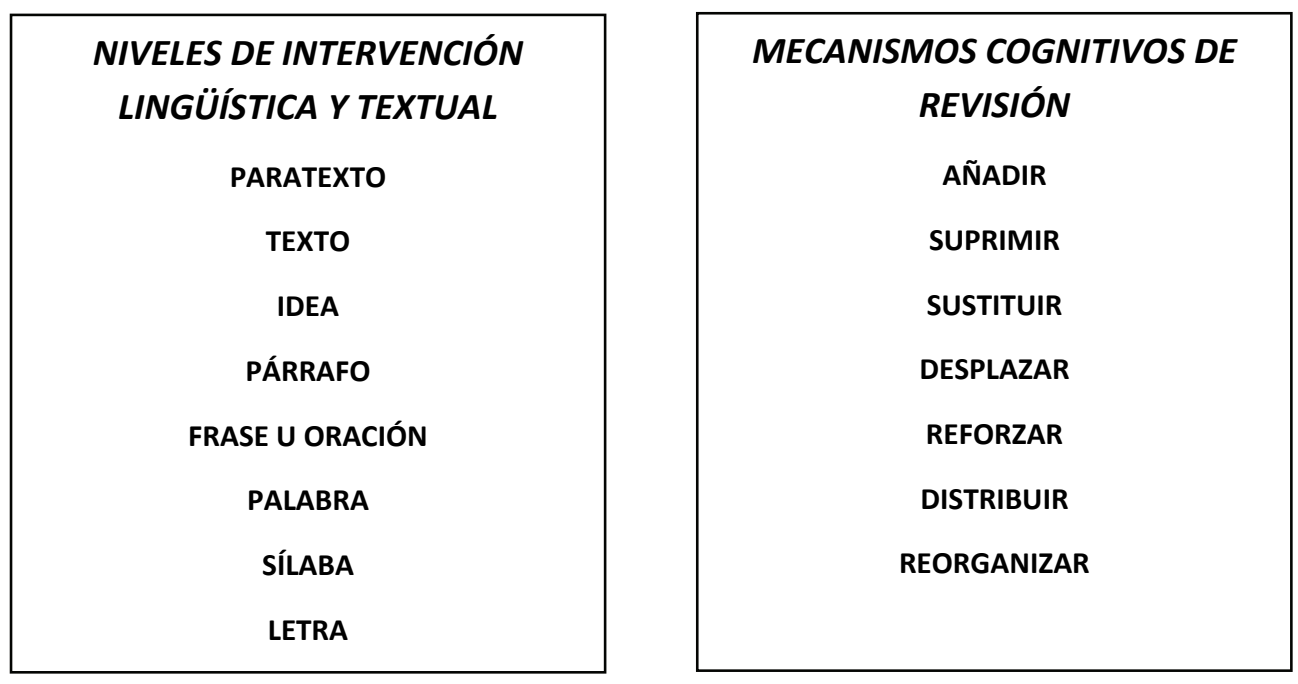

Cuadro 6.Cómo se revisa un texto.

En la segunda sesión, con las ayudas y las herramientas descritas y con la mediación de los cuatro estudiantes y el profesor, se lleva a cabo en el aula la revisión del texto, con el fin de mejorarlo y adecuarlo a lo que se propone.

Para la revisión y la reescritura de textos, se sigue el modelo CDO (ComparaciónDiagnóstico-Operación) de Bereiter y Scardamalia (1987). El proceso de revisión comienza con una comparación mental entre el borrador y el texto ideal. A continuación se realiza un diagnóstico para determinar las causas de los desajustes, errores, incoherencias, etc., y se interviene para subsanarlos, mediante la correspondiente propuesta de revisión y reescritura.

En el cuadro 7, se expone el procedimiento de revisión y reescritura seguido, así como algunas propuestas de modificación o reescritura formuladas por el grupo clase, a lo largo de la revisión del borrador, que fueron recogidas en el texto definitivo (Anexo 4).

\begin{tabular}{|l|l|l|}
\hline Mecanismo mental & \multicolumn{1}{|c|}{$\begin{array}{c}\text { Unidad } \\
\text { lingǘstica }\end{array}$} & \multicolumn{1}{c|}{ Propuesta de revisión/reescritura } \\
\hline Añadir & Paratexto & $\begin{array}{l}\text { Añadir pie de foto debajo de cada imagen que } \\
\text { acompaña al texto }\end{array}$ \\
\hline Añadir & Idea & $\begin{array}{l}\text { Añadir una definición del concepto de "terrafor- } \\
\text { mación", al comienzo del texto. }\end{array}$ \\
\hline Suprimir & Palabra & $\begin{array}{l}\text { En el séptimo párrafo de la segunda página, su- } \\
\text { primir "enteramente", en la frase "Todo ello es } \\
\text { enteramente correcto" }\end{array}$ \\
\hline Sustituir & Letra & Adoptar un criterio respecto de la escritura de \\
\hline
\end{tabular}




\begin{tabular}{|l|l|l|}
\hline & Frase & $\begin{array}{l}\text { terraformación, dióxido de carbono: aparecen con } \\
\text { mayúscula y con minúscula. } \\
\text { Se propone poner entre paréntesis CO2 }\end{array}$ \\
\hline Suprimir & Párrafos & $\begin{array}{l}\text { En el quinto párrafo, en la frase "La humanidad } \\
\text { necesitó 1600 años, 1650 para ser exactos", } \\
\text { suprimir "1650 para ser exactos". }\end{array}$ \\
\hline Sustituir & Párrafo & Unir los párrafos 7 y 8 \\
\hline Desplazar & Frases & $\begin{array}{l}\text { Desplazar el cuarto párrafo de la segunda hoja al } \\
\text { final de la introducción o incluso a la conclusión. }\end{array}$ \\
\hline Reforzar & $\begin{array}{l}\text { Añadir frases aclaratorias o explicativas sobre } \\
\text { conceptos técnicos, como "cinturón desértico". } \\
\text { Incluir "Ver gráfico" para establecer la relación } \\
\text { entre los datos del texto y el gráfico, como refe- } \\
\text { rencia intratextual. }\end{array}$ \\
\hline Reorganización & Párrafos & $\begin{array}{l}\text { Reforzar la idea del crecimiento demográfico, en } \\
\text { el quinto párrafo. }\end{array}$ \\
\hline Añadir & Frases & $\begin{array}{l}\text { Propuesta de reorganización: 4,2,1,3,6,5,7 } \\
\text { dad. }\end{array}$ \\
\hline
\end{tabular}

Cuadro 7. Procedimiento de revisión textual.

\section{Resultados}

Los datos recogidos de las revisiones del texto propuesto, realizadas por los estudiantes del grupo clase, se refieren, como indicamos más arriba, a los mecanismos mentales y a las unidades de lengua expresados para la revisión y la reescritura de textos.

La estadística que se presenta a continuación está basada en el orden en que se intervino en las unidades lingüísticas; es el siguiente:

\begin{tabular}{|l|c|c|c|c|c|c|c|c|}
\hline & Paratexto & Texto & Idea & Párrafo & Frase & Palabra & Sílaba & Letra \\
\hline Grupo 1 & 6 & & 6 & 3 & & & & \\
\hline Grupo 2 & 2 & 2 & 5 & 3 & 1 & 2 & & 1 \\
\hline Grupo 3 & 4 & & 2 & 1 & 6 & & & 1 \\
\hline Grupo 4 & & & & 4 & 4 & 2 & & 3 \\
\hline Grupo 5 & 1 & & 1 & & & 2 & & \\
\hline Grupo 6 & 1 & & & & 1 & & 1 & \\
\hline Grupo 7 & & & & & & 1 & & \\
\hline
\end{tabular}

Tabla 1. Unidades lingüísticas y textuales de la intervención. 
Gráfico 1. Representación del orden de intervención de las unidades lingüísticas

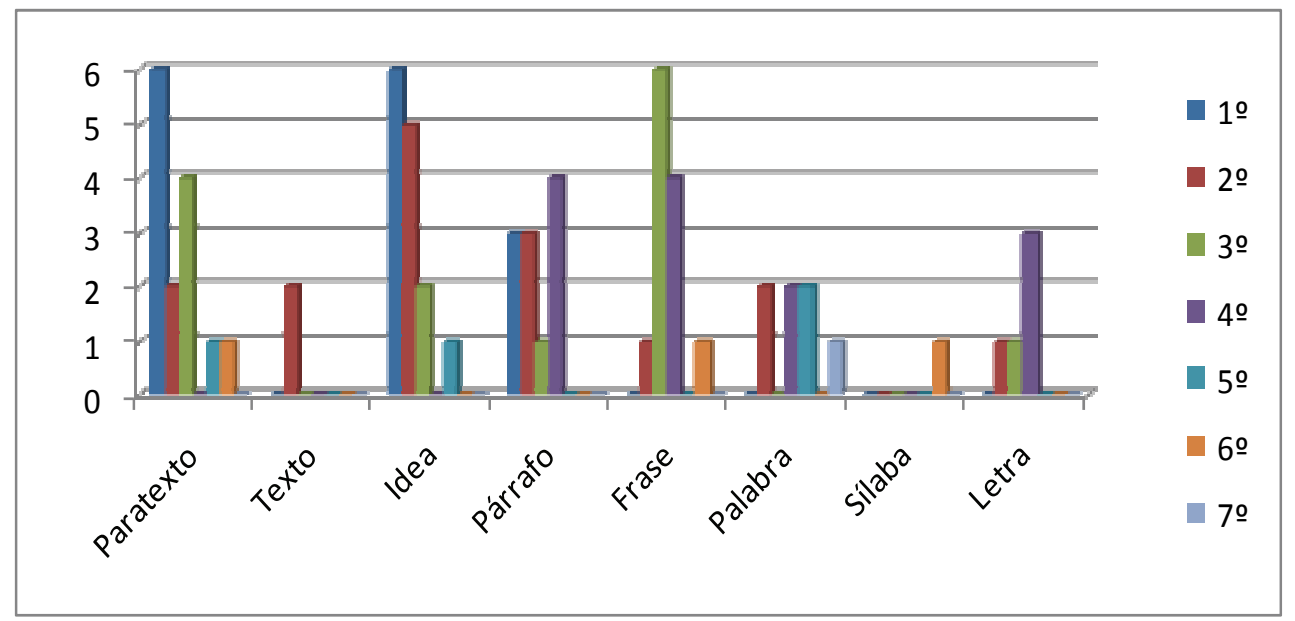

\begin{tabular}{|c|c|c|c|c|c|c|c|c|}
\hline & Paratexto & Texto & Idea & Párrafo & Frase & Palabra & Sílaba & Letra \\
\hline Añadir & 7 & & 10 & 2 & $2(+3)^{*}$ & & & \\
\hline Suprimir & 3 & & & 5 & 3 & 2 & & 4 \\
\hline Sustituir & 1 & & 1 & & 6 & 6 & & 2 \\
\hline Desplazar & & & & & & & & \\
\hline Reforzar & 5 & & 4 & & & & & \\
\hline Distribuir & & & & 2 & 1 & & & \\
\hline Reorganizar & & 1 & 1 & 5 & & & & \\
\hline
\end{tabular}

Tabla 2. Correspondencia entre los mecanismos mentales y las unidades lingüísticas. *(+3 puntos) hace referencia a los signos de puntuación. Se incluyen aquí porque pueden cambiar el significado de la frase. 
Gráfico 2. Representación de los mecanismos mentales y las unidades lingüísticas.

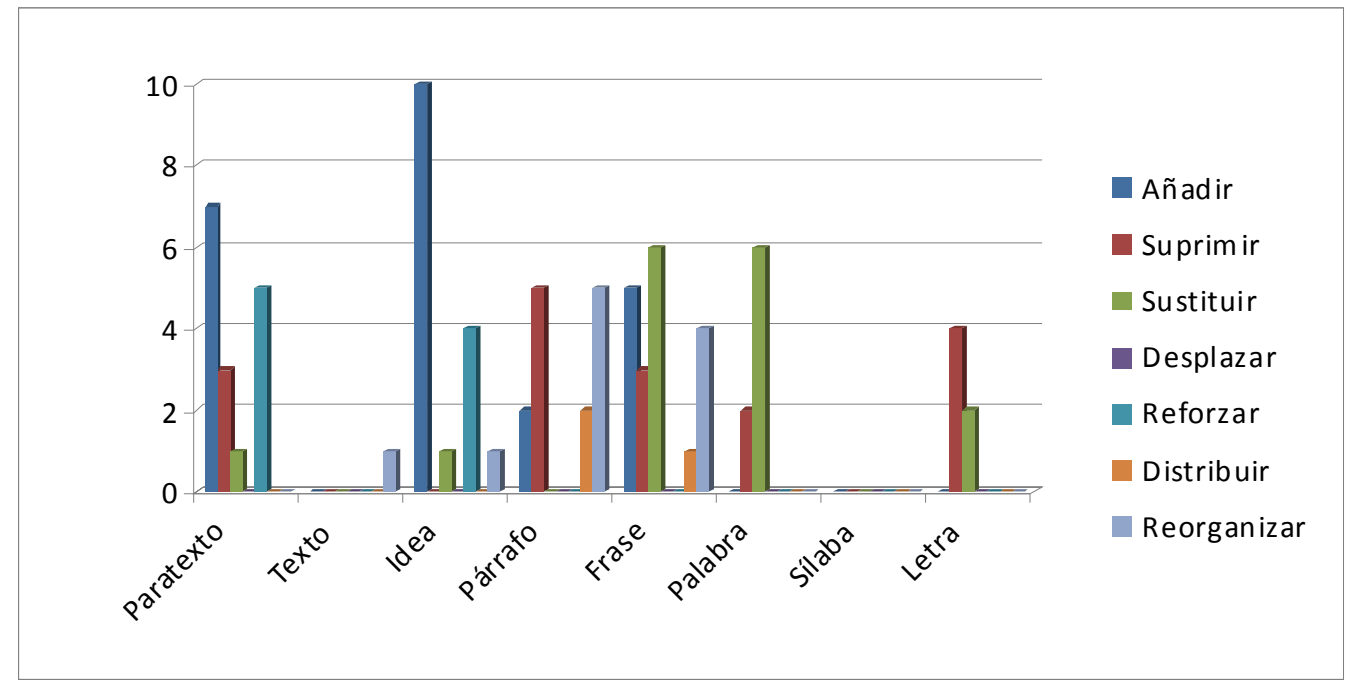

Puede verse en las dos tablas y en las dos últimos gráficos, en la revisión y la reescritura del texto destacan las funciones mentales de añadir, suprimir y reforzar las ideas y el paratexto, así como sustituir, reorganizar y reforzar las unidades relacionadas con el párrafo, la frase y la palabra. Las unidades correspondientes a la sílaba y a la letra tienen una presencia menor en estos textos producidos por universitarios.

\section{Discusión}

En la experiencia desarrollada, merece destacarse que las funciones mentales predominantes en las revisiones realizadas, así como las unidades lingüísticas en que más se interviene se basan en fenómenos relacionados con el género discursivo adoptado: la transmisión o divulgación de información, para el caso. A estos textos les caracteriza la intención de exponer y explicar información, con el fin de que el lector entienda bien lo que lee. Destacan las revisiones de párrafo, frase y palabra, que operan en el plano de la composición textual, mediante fenómenos, como: reformulación, sinonimia, monosemantismo, rigor, concisión y propiedad, regularidades del texto expositivo. Esto lleva consigo un recorrido o tránsito desde el caos del borrador al orden del que ha de gozar todo texto.

Es reseñable asimismo que las propuestas de sustitución de palabras, que sugerían determinados grupos, en la revisión y la reescritura del texto, confirman la idea de que los estudiantes entienden el proceso de revisión, principalmente, como una actividad léxica (sinonimia, antinomia, campo léxico, etc.). Esto, por otra parte, suscitó un debate interesante acerca de cuál ha de ser el grado de utilización del lenguaje técnico en un texto de divulgación, destinado a un público no especializado. Sin embargo, estos 
mismos estudiantes tienen más dificultad en detectar y reformular las repeticiones conceptuales y el texto como unidad, probablemente porque suelen subordinar las demandas de los problemas específicos del texto a la aplicación de reglas gramaticales y léxicas. Esto pone de manifiesto una concepción de la lengua predominantemente formal y normativa.

Las unidades correspondientes a la sílaba y a la letra tienen una presencia menor en estos textos producidos por universitarios, si bien es importante registrar estas unidades de lengua por la aplicación didáctica que tienen en la formación de maestros.

Es de destacar asimismo que la mayor parte de las revisiones realizadas por los diferentes grupos de clase fueron tenidas en cuenta en la redacción definitiva del texto, lo cual justifica la importancia de esta fase en la mejora del texto a lo largo del proceso de producción. Algunas propuestas de reescritura fueron desestimadas por el grupo de estudiantes que dirigió la actividad, por entender que el resultado era un texto distinto. Fue el caso, por ejemplo, de la reorganización de todos los párrafos del texto, como consta en el cuadro 7. Ello, a su vez, provocó no pocas discusiones acerca de la necesidad de optar por una u otra formulación textual, en función del contexto de producción de la escritura.

Con el fin de facilitar los mecanismos mentales de la revisión de textos en lo sucesivo, se propone reducir tales mecanismos a los siguientes: añadir, suprimir, sustituir, desplazar y reformular. Se entiende, por una parte, que todo desplazamiento lleva consigo distribuir y reorganizar la información; y, por otra parte, se incluye la reformulación como mecanismo cognitivo y textual, en los casos en que sea preciso reescribir fragmentos de texto, consecuencia de la unión de dos o más párrafos, ideas o frases, con el fin de aclarar mejor las ideas, de reorganizar una frase compuesta, etc.

\section{Conclusiones y proyección didáctica}

Esta actividad fomenta el trabajo integrado de las habilidades básicas del lenguaje (hablar, escuchar, leer y escribir) en el proceso de composición de un texto. Así, a lo largo del proceso, se escucha para hablar; se habla para escribir; se lee para saber; se lee para escribir; se lee para revisar; se escribe para revisar; se revisa para planificar; se reescribe para leer; y se lee, se habla y se escucha para exponer y defender en público.

Escribir en colaboración con otros muestra bien a las claras la complejidad que encierra la actividad de escritura, en cuanto que supone una oportunidad para aportar más ideas y más puntos de vista, exige capacidad de negociación en el acomodo al destinatario, la intención y el género discursivo, y para progresar en la redacción. Ello enriquece la actividad de composición de textos, tanto en la adquisición de información, como en el conocimiento del género en cuestión.

Consecuencia didáctica de lo anterior es la importancia de ayudar a los alumnos para que visualicen fácilmente las operaciones mentales y los niveles lingüísticos en que han de intervenir para mejorar el texto y tomar conciencia progresivamente de la complejidad (y del provecho intelectual y social, también) que encierra revisar y reescribir 
textos. Para ello, es importante diseñar herramientas que faciliten la dificultad de esta tarea. Esto favorecerá la interiorización y la automatización de las estrategias que requiere esta habilidad, así como la progresiva autonomía del individuo en la competencia en escritura.

El papel del profesor consiste en ser un guía permanente, que media en el proceso, asesora en el desarrollo de la producción del texto y proporciona la información y las estrategias y ayudas necesarias para redactar, exponer y, en su caso, defender en público el contenido del texto.

Todo ello hace, pues, que la composición de textos sea una habilidad compleja, y que, por tanto, haya que enseñarla y aprenderla en todos los niveles y en todas las materias del sistema educativo, con la ayuda de herramientas que construyan el andamiaje necesario que facilite la producción del texto.

La principal limitación de este trabajo es que se basa en una experiencia que muestra una actividad ceñida a un grupo de clase de estudiantes universitarios, con la necesaria proyección profesional (didáctica), pensada para ser replicada en las aulas de finales de Educación Primaria. Se puede afirmar, sin embargo, que la experiencia puede llevarse a cabo también en cursos de Educación Secundaria Obligatoria.

Ahora bien, esta experiencia merecería ser generalizada con muestras más amplias de alumnos de Educación Obligatoria, y de estudiantes del Grado en Magisterio y del Máster de Formación del Profesorado de Secundaria, con el fin de comprobar qué mecanismos cognitivos y qué unidades lingüísticas predominan en la revisión de textos, de modo que se puedan introducir elementos de ayuda y reflexión, y así poder establecer la consiguiente metodología de revisión de estos textos. 


\section{Anexo 1 \\ $2^{\text {a }}$ FASE: DOCUMENTACIÓN Y SELECCIÓN DE INFORMACIÓN \\ PRODUCTO:}

Tema: La Terraformación

Destinatario: Público no especializado

Intención: Despertar el interés por un tema

Dar cuenta de lo que se sabe

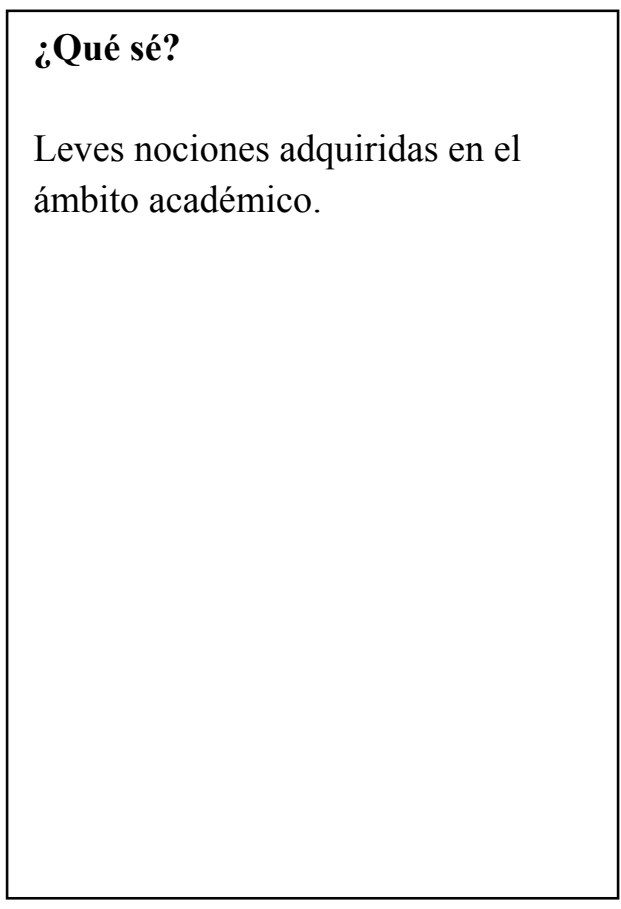

\section{¿Qué quiero saber?}

- Situación actual de la Terraformación en el ámbito científico.

- Posibilidades de puesta en práctica (económicas, morales, tecnológicas...)

\section{¿Dónde buscarlo?}

- Departamento de Ciencias Naturales (UCM).

- Portales de las agencias ESA, NASA...

- Wikipedia

- Artículos en la web. 
Anexo 2

\author{
El texto expositivo y su escritura \\ Preguntas que te ayudarán a redactar el texto
}

- Antes de escribir:

- ¿Has pensado en los lectores de tu texto?

- ¿Has dejado claro la intención que persigues con el texto?

- ¿Tienes claro cómo son los textos que se usan para exponer o informar?

- ¿Te has preguntado qué sabes sobre el tema y qué quieres saber sobre ese mismo tema?

- ¿Has pensado dónde se puede buscar información sobre ese mismo tema?

- ¿Te has preguntado cómo lo vas a difundir (Medio, soporte, etc.)?

- ¿Tienes claro si el texto va a ser expuesto oralmente? ¿Dónde: en clase, o ante otro auditorio?

\title{
- Durante el proceso de escritura:
}

- ¿Has buscado y manejado documentación suficiente?

- ¿Has utilizado algún modelo o molde de organización de la información?

- ¿Has tenido en cuenta los organizadores del texto (títulos y subtítulos) y elementos ilustradores, tales como: mapas, planos, gráficos, esquemas, mapas conceptuales, etc.?

- ¿Has tenido presente que tu texto ha de ser coherente, estar cohesionado, ser adecuado, sintácticamente correcto e informativamente rico?

- ¿Consideras que utilizas el léxico con precisión?

- ¿Has ido revisando el texto mientras lo ibas escribiendo?

\section{- Después de haber escrito el texto:}

- ¿Has revisado el texto para localizar aciertos y errores, antes de darlo por definitivo?

- ¿Has consultado dudas (a compañeros y a profesores) y has anotado las modificaciones para mejorar el texto?

- ¿Has buscado y te has asesorado con tu profesor acerca del formato más idóneo para editar el texto?

- ¿Has preparado la presentación oral? 


\section{Anexo 3}

\section{PLANTILLA DE REVISIÓN DE TEXTOS EXPOSITIVOS}

1. ¿Se distingue con claridad la intención del texto?

- Sí.

- No.

Razona tu respuesta.

2. ¿Se identifica el destinatario?

- Sí.

- No.

¿A quién crees que podría estar dirigido?

3. ¿Cuál es el subtipo predominante?

- Clasificación-tipología.

- Problema-solución.

- Pregunta-respuesta.

- Descripción-definición.

- Causa-consecuencia.

4. ¿Se observa un predominio de los datos o hechos sobre las opiniones?

- Sí.

- No.

5. ¿Está el tema contextualizado mediante definiciones, recorrido histórico,...?

- Sí.

- No.

6. ¿Se presentan con claridad las ideas principales?

- Sí.

- No.

¿Cuáles son?

7. ¿Predominan los tiempos verbales propios de la exposición (Presente con valor atemporal y/o formas no personales del verbo)?

- Sí.

- No.

8. ¿Qué adjetivación predomina?

- Pospuesta.

- Antepuesta.

9. ¿Están los argumentos fundamentados a partir de la opinión de expertos, datos bibliográficos u otros recursos?

- Sí.

- No.

Razona la respuesta.

10. Se realizan explicaciones a través de:

- Ejemplificaciones.

- Enumeraciones.

- Comparaciones.

- Dos puntos introductorios de explicación.

- Paréntesis aclaratorios.

- Que explicativo.

- Aposiciones explicativas. 
11. ¿Resulta el texto informativo?

- Sí.

- No.

12. ¿Está exento de contradicciones internas?

- Sí.

- No.

13. ¿Se contempla la estructura clásica de problema, selección y resumen

- Sí.

- No.

Razona la respuesta.

14. ¿Se presentan los párrafos ordenados de forma lógica?

- Sí.

- No.

15. Se observan disfunciones en:

- Utilización de frases hechas.

- Imprecisión y vaguedad en el uso del os términos.

- Omisiones indebidas.

- Pleonasmos o redundancias.

- Uso de gerundios.

- Construcción lógico-sintáctica de la frase (S.V.O.)

16. ¿Tiene faltas de concordancia de orden nominal y/o verbal?

- Sí.

- No.

17. Tipos de faltas ortográficas:

- Cambio de letras.

- Tildes.

- Hiposegmentación o hipersegmentación.

- Siglas y abreviaturas.

- Otras (especificar).

18. ¿Proporciona la puntuación claridad al texto?

- Sí.

- No.

Señala los usos inadecuados de:

- Coma.

- Punto y coma.

- Punto seguido.

- $\quad$ Punto y aparte.

- Dos puntos.

- Signos de interrogación.

- Otros.

19. ¿Hay referencia explícita a las fuentes de documentación manejadas?

- Sí.

- No.

20. ¿Es el texto científico?

- Sí.

- No.

Justifica los motivos. 


\section{Anexo 4. Texto definitivo}

\section{Terraformación: Condiciones de posibilidad de una ciencia incipiente. Introducción.}

La humanidad ha puesto rumbo hacia una fase crítica de su desarrollo. En nuestro horizonte se dibujan el agotamiento de los recursos y la explosión demográfica y, con ellos, conflictos de consecuencias imprevisibles.

La mayoría de las personas son conscientes de la necesidad de establecer un equilibrio entre el hombre y la naturaleza que le rodea, pero, mientras algunos confían en las fuerzas autorreguladoras de la naturaleza, otros fundan su esperanza en la razón y su capacidad para una planificación inteligente.

Aunque no es posible encontrar una coincidencia de opiniones sobre la cuestión de si sería deseable y cómo una mejora para nuestra especie, es forzoso reconocer como una meta necesaria la supervivencia de la descendencia humana.

Por tanto, nuestro comportamiento será el apropiado si nuestras acciones aseguran dicha supervivencia.

¿Cuál es el núcleo del problema?

El economista británico Thomas Robert Malthus advirtió ya en 1798 que el crecimiento demográfico incontrolado lleva a que la población se multiplique por encima del límite de tolerancia del espacio vital, de manera que, al final, la base de la alimentación acaba siendo insuficiente para sustentar a los hombres. En esencia, esta teoría demográfica ha acreditado su validez: el crecimiento de la humanidad es exponencial.

Si tomamos como base para el cálculo los 250 millones de personas que habitaban la Tierra en torno al nacimiento de Cristo, la humanidad necesitó 1650 años para duplicar esa cifra; otros 200 años para volverla a doblar (año 1850); cien años más para doblarla por tercera vez (1950), y sólo 26 años para la cuarta, que elevó la población del mundo a más de 4000 millones. En las últimas décadas la tasa de crecimiento anual, que durante milenios rondó el 0.2 por ciento, pero que en la década de los 70 del siglo pasado anduvo en torno al 2 por ciento, ha comenzado a reducirse parcialmente, a pesar de ello, el planeta está, en la actualidad, poblado por más de 6000 millones de personas (Ver gráfica 1). 


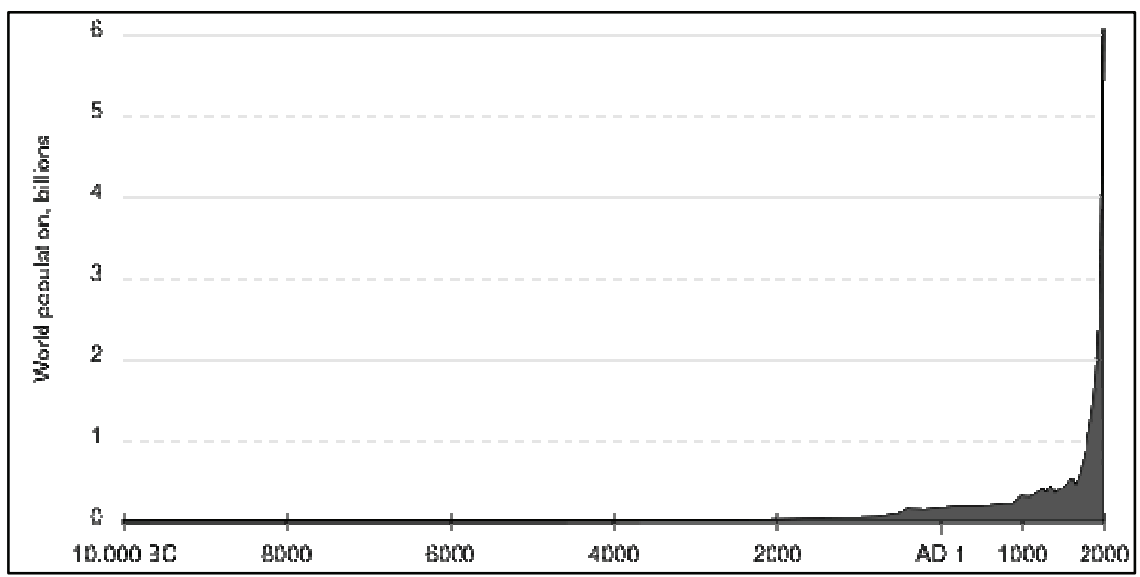

Gráfica 1. Crecimiento de la población mundial

Debido a este incremento, los recursos derivados del petróleo y las materias primas son casi insuficientes para proporcionar a todos estos habitantes unos niveles de vida aceptables.

El hombre, a día de hoy, extrae reservas de toda la biosfera. Obtiene materias primas vegetales, animales y minerales del mundo entero; y explota, sobre todo, combustibles fósiles (carbón y petróleo) provocando así cambios globales en la naturaleza.

El aumento progresivo de los contenidos de CO2 en la atmósfera, junto con los gases de origen industrial, producen un recalentamiento de la Tierra, convirtiendo el efecto invernadero en un verdadero problema medioambiental. Se contempla la posibilidad de la fusión de los casquetes polares, lo que puede provocar una severa inundación de las tierras bajas y de las ciudades costeras, así como una ampliación de la extensión del cinturón desértico.

La sociedad moderna, a pesar de sus logros científicos y tecnológicos, ha alumbrado nuevos temores y ha favorecido un claro empeoramiento de la calidad de vida. Entendida ésta tanto desde el ámbito ecológico, como desde el punto de vista interpersonal. Pero el hombre no sólo tiene la capacidad de adaptarse a condiciones cambiantes, sino también la facultad de fijarse metas y organizar, según ellas, un nuevo orden de prioridades. Esto abre posibilidades desconocidas hasta ahora, pero también riesgos. La determinación de objetivos, sea cuál sea su naturaleza, establece el curso de la evolución científica, $\mathrm{y}$, en definitiva, de la propia evolución humana.

Las cosas podrían haber acaecido de cualquier otra manera, pero así han transcurrido. Todavía es pronto para realizar diagnósticos desesperados, aunque no para señalar que el problema ha desbordado el perímetro de la crisis menor y que nos enfrentamos a un futuro incierto. 
Todo ello es correcto, pero también es cierto que nuestro comportamiento y el hecho de idear unas metas, abren camino a la investigación y que tenemos la posibilidad de configurar decisivamente nuestro futuro, imaginando los objetivos correspondientes. Ésta es la imagen tradicional de cómo progresa la ciencia.

Sin embargo, por mucho que imaginemos la mejor de las adaptaciones a unas determinadas condiciones ambientales, hostiles en cada caso concreto, nos será difícil poder objetar contra estos ambiciosos proyectos algo más que la constatación del miedo a lo desconocido, al porvenir, precisamente porque estamos todavía muy apegados a la vida terrestre.

Y aún así, si la destrucción total del hábitat nos condujera a una obligatoria terraformación planetaria (conjunto de procesos orientados a la intervención de un planeta, luna $\mathrm{u}$ otro cuerpo celeste para recrear en este las condiciones óptimas para la vida terrestre, a saber, una atmósfera y temperatura adecuadas, y la presencia de agua líquida), no podríamos contestar con una inmovilidad conservadora: deberíamos, por el contrario, haber ultimado las fases experimentales de la readaptación.

Por ello, el método de la ciencia actual, y en especial el de la terraformación, pasa por la construcción de modelos matemáticos. Dichos modelos sólo corresponden a la realidad que modelan con grandes dosis de aproximación y simplificación y, a pesar de ello, nos permiten comprender una realidad de otro modo incomprensible: formular preguntas precisas, utilizar en la computación todas las posibilidades del cálculo matemático y obtener así, finalmente, respuestas aproximadamente correctas.

Más vale una aproximación computable, que una verdad absoluta incomputable. Más vale el arduo progreso de la ciencia real, que la fatua añoranza de un saber imposible.

\section{Notas}

Agradezco la participación de los cuatro estudiantes, cuyos nombres son: Aurora Agulló Fernández, Carlos Alonso Aguirre, Sara Cubero Mateos y Andrés Romero Bravo.

\section{Referencias bibliográficas}

ALLAL, L., L. CHANQUOY AND P. LARGY (Eds.) (2004). Revision. Cognitive and Instructional Process. Norwell, Massachusetts: Kluwer Academic Press.

ÁlVAREZ, T. (2001). Textos expositivo-explicativos y argumentativos. Barcelona: Octaedro.

--- (2005). Didáctica del texto en la formación del profesorado. Madrid: Síntesis.

--- (2010). Competencias básicas en escritura. Barcelona: Octaedro.

--- y R. Ramírez (2006). "Teorías o modelos de producción de textos en la enseñanza y el aprendizaje de la escritura". Didáctica (Lengua y Literatura), 18, 29-60. 
BARTON, D. AND R. IVANIC (Eds.) (1991). Writing in the Community. London: Sage.

BAZERMAN, CH. AND H. S. WIENER (2003). Writing Skills Handbook. Boston: Houghton Mifflin Company.

BAZERMAN, CH. et al. (2005). Referente Guide to Writing Across the Curriculum. Indiana: Pador Press.

--- (Eds.) (2010). Traditions of Writing Research. New York: Routledge

BEREITER, C. Y M. SCARDAMALIA (1987). The Psychology of Written Composition, NJ Erlbaum, Hillsdale.

CAMARGO, Z., G. URIBE Y M. A. CARO (2011). Didáctica de la comprensión y producción de textos académicos. Armenia, Colombia: Universidad del Quindío

CAMPS, A. (Comp.) (2003). Secuencias didácticas para aprender a escribir. Barcelona: Graó.

CARLINO, P. (2006). Escribir, leer y aprender en la universidad. Una introducción a la alfabetización académica. Buenos Aires: Fondo de Cultura Económica.

CASTELLÓ, M. (Coord.) (2007). Escribir y comunicarse en contextos científicos y académicos. Conocimientos y estrategias. Barcelona: Graó.

DIDACTEXT, GRUPO (2003). Modelo sociocognitivo, pragmalingüístico y didáctico para la producción de textos escritos. Didáctica (Lengua y Literatura), 15, 75-102.

--- (2005). Los procesos de escritura y el texto expositivo en la mejora de la competencia escrita de los escolares de Educación Primaria, Madrid, Editorial Complutense.

DOLZ, J., M. C. ROSAT Y B. SCHNEUWLY (1990). Elaboration et évaluation de deux séquenles didactiques relatives à trois types de textes. Le français aujourd'hui, 92, 37-46.

KLEIN, P. D. \& KIRKPATRICK, L. C. (2010). A framework for content area writing: Mediators and moderators, Journal of Writing Research, 2 (1), 1-46.

MACARTHUR, CH., S. GRAHAM AND J. FITZGERALD (Eds.) (2006). Handbook of Writing Research. New York: The Guilford Press.

MILLET, S. (Ed) (2009). The Norton Book of Composition Studies. New York : Noton \&Compan

PETILLON, S. Y F. GANIER (2006). "La révision de texte: méthodes, outils et processus". Langages, 164. (Monográfico).

SCHNEUWLY, B. (1992). La concepción vygotskiana del lenguaje escrito. Comunicación, Lenguaje y Educación, 16, 49-59.

SOMMERS, N. (2009). Revision Strategies of Students Writers and Experienced Adult Writers. En Susan Millet (ed). The Norton Book of Composition Studies. New York : Noton \& Company, 323-332.

VAN WAES, L., M. LEIJTEN AND T. QUINLAM (2010). Reading Turing sentence composing and error correction: A multilevel análisis of the influences of task complexity. Reading and Writing, 23, 803-834. 
VYGOTSKY, L. S. (1932). Pensamiento y lenguaje, La Plèyade, Buenos Aires, 1987.

--- (1978). El desarrollo de los procesos psicológicos superiores. Barcelona: Crítica.

Correspondencia con el autor

Teodoro Álvarez Angulo

Dpto. Didáctica de la Lengua y la Literatura Facultad de Educación

Universidad Complutense de Madrid

e-mail: talang@edu.ucm.es 